\title{
Exploiting 2-Dimensional Source Correlation in Channel Decoding with Parameter Estimation
}

\author{
Muhammad Izzat Amir Mohd Nor, Mohd Azri Mohd Izhar, Norulhusna Ahmad, Hazilah Md. Kaidi
}

Razak School of UTM in Engineering and Advanced Technology, Universiti Teknologi Malaysia, Malaysia

\begin{tabular}{l}
\hline \hline Article Info \\
\hline Article history: \\
Received Mar 9, 2018 \\
Revised Jun 25, 2018 \\
Accepted Jul 2, 2018 \\
\hline
\end{tabular}

\section{Keyword:}

Bahl-cocke-jelinek-raviv algorithm

Baum-welsh algorithm

Joint source channel coding

Markov chain

Parameter estimation

\begin{abstract}
Traditionally, it is assumed that source coding is perfect and therefore, the redundancy of the source encoded bit-stream is zero. However, in reality, this is not the case as the existing source encoders are imperfect and yield residual redundancy at the output. The residual redundancy can be exploited by using Joint Source Channel Coding (JSCC) with Markov chain as the source. In several studies, the statistical knowledge of the sources has been assumed to be perfectly available at the receiver. Although the result was better in terms of the BER performance, practically, the source correlation knowledge were not always available at the receiver and thus, this could affect the reliability of the outcome. The source correlation on all rows and columns of the 2D sources were well exploited by using a modified BahlCocke-Jelinek-Raviv (BCJR) algorithm in the decoder. A parameter estimation technique was used jointly with the decoder to estimate the source correlation knowledge. Hence, this research aims to investigate the parameter estimation for 2D JSCC system which reflects a practical scenario where the source correlation knowledge are not always available. We compare the performance of the proposed joint decoding and estimation technique with the ideal 2D JSCC system with perfect knowledge of the source correlation knowledge. Simulation results reveal that our proposed coding scheme performs very close to the ideal 2D JSCC system.
\end{abstract}

Copyright $@ 2018$ Institute of Advanced Engineering and Science. All rights reserved.

\author{
Corresponding Author: \\ Mohd Azri Mohd Izhar, \\ UTM Razak School of Engineering and Advanced Technology, \\ UTM Kuala Lumpur, Level 7, Razak Tower, Jalan Sultan Yahya Petra, 54100 Kuala Lumpur, Malaysia. \\ Email: mohdazri.kl@utm.my
}

\section{INTRODUCTION}

Many improvements were implemented to channel coding including turbo coding which was introduced in 1993 [1]. The advantages of the turbo codes include high reliability and near to Shannon's capacity performance and hence, make turbo codes to be well adopted in various applications [2]-[4]. Shannon's separation theorem states that an optimal performance in separate source and channel coding system can be achieved if channel coding and source coding are optimal with zero delay [5]. However, it is impossible to get the finest performance with zero delay as the restriction on latency definitely occurs in practical applications. Joint Source and Channel Coding (JSCC) has been introduced by making use of the residual redundancies left in source encoders to improve the error correction capability of a channel code [6]-[8].

Recent research of [9], [10] have introduced 2-Dimensional (2D) source correlation for a JSCC system was exploited and utilized in the system design to improve the Bit Error Rate (BER) performance. However, these studies assumed that the source correlation knowledge is perfectly known at the receiver [9]-[11]. This assumption improves the performance but it affects the reliability of the information of source correlation to be exploited at the receiver. Practically, source correlation knowledge is unknown and needs to be estimated. Thus, many researchers have taken up this issue and developed various parameter estimation 
techniques to estimate the source correlation at the receiver to ensure the performance is improved and applicable in real practices [12], [13].

Several methods of parameter estimation have been presented in various studies based on a Hidden Markov Model (HMM) such as the Baum-Welch Algorithm (BWA) [14]-[20]. This enables the decoder to utilize the estimated statistics of the source correlation knowledge in channel decoding to improve the system performance. In this paper, we propose 2D JSCC system with BWA-based estimation technique to estimate the source correlation knowledge at the channel decoder on the non-HMM model to get the performance as close as possible to the ideal system. The proposed design is then compared with the existing 2D JSCC system with the perfect assumption on source correlation knowledge or known as the ideal 2D JSCC system [9] and another technique of parameter estimation exploiting the extrinsic information gleaned from the decoders adopted from [11].

This paper has been organised in the following way. The related works of parameter estimation techniques on channel decoding and the mechanism of BWA are discussed in Section 2. The proposed model on the coding scheme is presented in Section 3. Section 4 deliberates on the difference between BER performance from the proposed coding scheme and existing work. Finally, Section 5 draws upon the entire thesis, gives a conclusion and identifies areas for further research.

\section{RELATED WORKS ON PARAMETER ESTIMATION TECHNIQUES}

\subsection{Parameter estimation on turbo decoder}

Several attempts have been made previously to describe the parameter estimation of Markov model at the Turbo decoder in [15], [16]. These approaches have known to be complex due to the essence of building the supertrellises combining both constituent encoders and the HMM models. The modification on the decoder [17] has been done to improve the performance while the simplified method for modifying a decoder has been described in [18] to reduce its complexity while maintaining the same performance as in [16]. The parameter estimation has been refined in [19] to enhance the connection between BWA as the parameter estimation technique and Turbo decoder. BWA is implemented in the first iteration in order to have an initial estimation of Hidden Markov source parameters. The correlation pattern is considered and characterized by HMM [19]. The expected number of transitions from state to state that generate correlation pattern $\mathrm{v}$ are represented by the product of the branch probability and the number of trellis transitions. Hence, making it easy to estimate the expected state transition. Anwar, in [20], suggested that the stability and efficient exploitation of BWA can be improved by using a forward-backward algorithm or the uni-directional algorithm.

\subsection{The simplified Baum-Welsh algorithm}

The potential algorithm associated with the learning problem of an HMM is the BWA which has been developed in 1970 [21] and also known as Forward-Backward algorithm which is considered as the unique case of the Expectation-Maximization or EM algorithm [22]. In the learning problem, the parameters of an HMM denoted as are developed and adjusted depending on the applications given an observation sequence $\mathrm{O}$ and the set of possible states $\mathrm{Q}$. Meanwhile, for the non-HMM considered in this work, we only used one parameter to be estimated to reduce the complexity of the system while maintaining its peak performance. In this method, only transition probabilities are used to estimate the source statistics in the decoder. The equation of transition probability is adopted from the previous work done by Garcia-Frias and Villasenor in [19]. The BWA is generally performed by calculated the initial estimation for the probabilities, and iteratively uses the information from the estimated value to perform better estimation upon the probabilities on the next iteration. The forward variables can be given as:

$$
\alpha_{t}(i)=P\left(o_{1}, o_{2}, \ldots, o_{t}, q_{t}=i \mid \lambda\right),
$$

where is forward variable, and is the partial observation sequence while the backward variable can be stated as:

$$
\beta_{t}(i)=P\left(o_{t+1}, o_{t+2}, \ldots, o_{t} \mid q_{t}=i, \lambda\right),
$$

where $\beta_{t}(i)$ is the probability of the partial sequence $o_{t+1}, o_{t+2}, \ldots, o_{t}$ at the current state $i$. 
The forward and backward variables in Equation (1) and Equation (2), respectively can be implemented to initialize the transition probability. The transition probability can be estimated as

$$
\hat{a}_{i j}=\frac{\text { expected number of transitions from state } i \text { to state } j}{\text { expected number of transitions from state } i}
$$

In order to calculate the numerator of, the joint probability of being in state at time and state at time denoted as is defined as:

$$
\xi_{t}(i, j)=P\left(q_{t}=i, q_{t+1}=j \mid O, \lambda\right)
$$

where the observation sequence and are given. The equation also can be written as:

$$
\xi_{t}(i, j)=\frac{P\left(q_{t}=i, q_{t+1}=j \mid O, \lambda\right)}{P(O \mid \lambda)}
$$

By using the forward and backward variables, the final equation of can be modeled as

$$
\xi_{t}(i, j)=\frac{\alpha_{t}(i) a_{i j} b_{j}\left(o_{t+1} \beta_{t+1}(j)\right)}{\alpha_{T}(q F)},
$$

where is the final state.

Thus, the numerator of the transition probability can be calculated as a summation of for all $\mathrm{t}$ while the denominator of which is the summation of all the expected number of transitions in state can be denoted The final formula for can be stated as

$$
\hat{a}_{i j}=\frac{\sum_{t=1}^{T-1} \xi_{t}(i, j)}{\sum_{t=1}^{T-1} \sum_{k=1}^{N} \xi_{t}(i, k)} .
$$

In summary, the estimation of the transition probability can be re-estimated using Equation (7) again to exploit the previous estimated value. The re-estimation process is considered as the basis of the iterative forward-backward algorithm.

\section{SYSTEM DESIGN}

Figure 1 illustrates the top-level design of the proposed communication system. The source natural redundancy was considered in this study for simplicity and generalization purposes as there is no source encoder employed in the proposed system. The source is a 2D binary source having a 2D source correlation. The 2D source is converted into a 1D sequence represented by a vector $\mathbf{u}$ before being encoded by a channel encoder to output the encoded sequence $\mathbf{c}$. Then, the encoded sequence $\mathbf{c}$ is modulated by a BPSK modulator to generate modulated output sequence $\mathbf{g}$ before being transmitted over an AWGN channel. During the modulation process, the binary encoded sequence is transformed into a signal form that can be physically transmitted, i.e., bit 0 maps to a signal amplitude -1 and bit 1 maps to a signal amplitude 1 .

In a real environment, distortion and noise always be the factors that disturb the received signal at the receiver. Most commonly used and simple model to denote the channel behaviors of a communication system is the Additive White Gaussian Noise (AWGN), which is due to the thermal noise produced by the electronic hardwares and devices. In this model, the independent Gaussian noise signals with zero mean $n$ and variance are added to the transmitted signal $\mathrm{g}$. The energy of the transmitted signal is normalized to 1 and the examined energy per bit to noise power spectral density ratio, is used to measure the channel quality. The signal will be distorted and sent to the receiver as $\mathrm{z}=\mathrm{g}+\mathrm{n}$. At the receiver, the received signal sequence $\mathrm{z}$ is demodulated by a BPSK demodulator to generate an output sequence $\mathrm{r}$. The a posteriori information of the

Exploiting 2-Dimensional Source Correlation in Channel Decoding ... (Muhammad Izzat Amir Mohd Nor) 
source correlation knowledge is estimated during channel decoding using the proposed parameter estimation technique. The new estimated value of source correlation knowledge is exploited at the decoder before outputting the decoded binary sequence. The detail of the proposed transmitter and receiver model will be presented in the following sections.

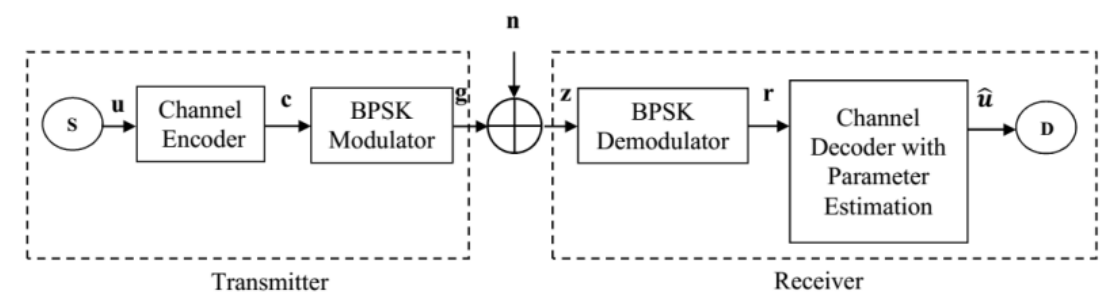

Figure 1. General view of the proposed JSCC design

\subsection{Transmitter design}

The proposed encoder employs two channel codes that are serially concatenated to each otherwhich are Turbo Single Parity Check Code (TSPCC) [23], [24] and a rate-1 Recursive Systematic Convolutional Code (RSCCs) [25], [26]. The TSPCC acts as an outer code and comprises a concatenation of multiple SPCCs arranged in parallel with interleavers separating the SPCCs. A rate-1 RSCC is employed as the inner code, to improve the performance of the proposed system and to remove the high error-floor problem encountered by TSPCC [23], [24].

Figure 2 illustrates the block diagram of the transmitter model for the proposed JSCC system exploiting 2D source correlation. The 2D sources of is generated by $\mathrm{S}$. The research target is to come out with an estimation technique for the 2D correlation parameters that can work well with the decoder of [9], thus the same design is used for the transmitter model. Two component encoders for TSPCC, and are considered. The interleaver, is a block interleaver that is used to arrange the source sequence to a sequence corresponding to the different direction of the source correlation denoted by. A TSPCC uses SPCCs as its component codes and it is known that SPCCs are simple codes that only generate a single parity check bit for any length of information bits. The advantage of this code is the flexibility in working with any length of information bits and it can operate at a very high coding rate. The parity bit sequences, and and source sequences are multiplexed after the source sequences have been encoded by the component codes. This joined sequence $\mathrm{w}$ is then rearranged by a random interleaver before passed through another encoding process by. The final encoded sequence $\mathrm{c}$ is modulated using a BPSK modulator before being transmitted over an AWGN channel.

\subsection{Receiver design}

The detailed receiver model of the proposed JSCC system is shown in Figure 3. The signal from the channel is received and demodulated to generate an output sequence, r. By using Bahl-Cocke-Jelinek-Raviv (BCJR) algorithm in, the sequence $r$ is decoded via Maximum A Posteriori (MAP) decoding process [27]. Soft information in Log-Likelihood Ratio (LLR) form is used while performing the decoding process. The a priori LLR of coded bit, at a time index $t$, can be computed as:

$$
L_{a}\left(w_{t}\right)=\ln \frac{P\left(w_{t}=1\right)}{P\left(w_{t}=0\right)}
$$

where is the probability that has the value of 1 , while is the probability of holds the value 0 . The extrinsic LLR, that is generated from is de-interleaved using before fed into and. The two component decoders correspond to the decoding of the horizontal and vertical direction source sequence. Modified BCJR algorithm is employed to exploit the source correlation throughout the decoding process [9]. Each component decoder yields a posteriori and extrinsic LLRs outputs. The a posteriori are then sent to the parameter estimation technique block which employs the BWA given in Equation (7) to estimate the transition probabilities where $\hat{p}_{0}^{D_{1}}=\hat{p}_{0}^{D_{2}}=\hat{a}_{00}$ and $\hat{p}_{1}^{D_{1}}=\hat{p}_{1}^{D_{2}}=\hat{a}_{11}$. The extrinsic LLRs from both activated component decoders that correspond to the source sequence $\mathrm{u}$ are summed up and multiplexed with all the extrinsic LLRs corresponding to the parity bit sequences. The combined sequence is then interleaved by $\Pi_{\text {in }}$ before feedback to $C_{\text {in }}^{-1}$. 
This process is repeated for a number of iterations. After the final iteration, the a posteriori $L_{a p p}^{2}$ from $C_{2}^{-1}$ is deinterleaved by $\Pi_{\mathrm{in}}^{-1}$. Hard decisioning is made to the a posteriori LLRs output $L_{a p p}^{2}$ to generate the decoded bits sequence $\hat{u}$.

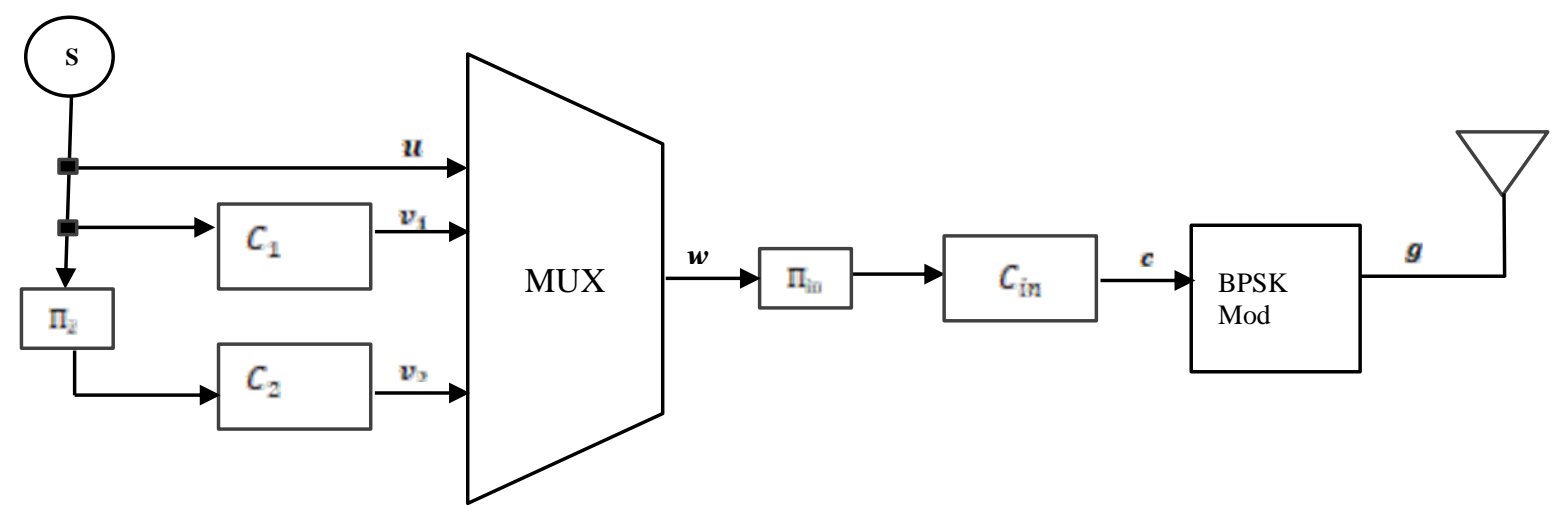

Figure 2. Proposed transmitter JSCC system exploiting 2D source correlation

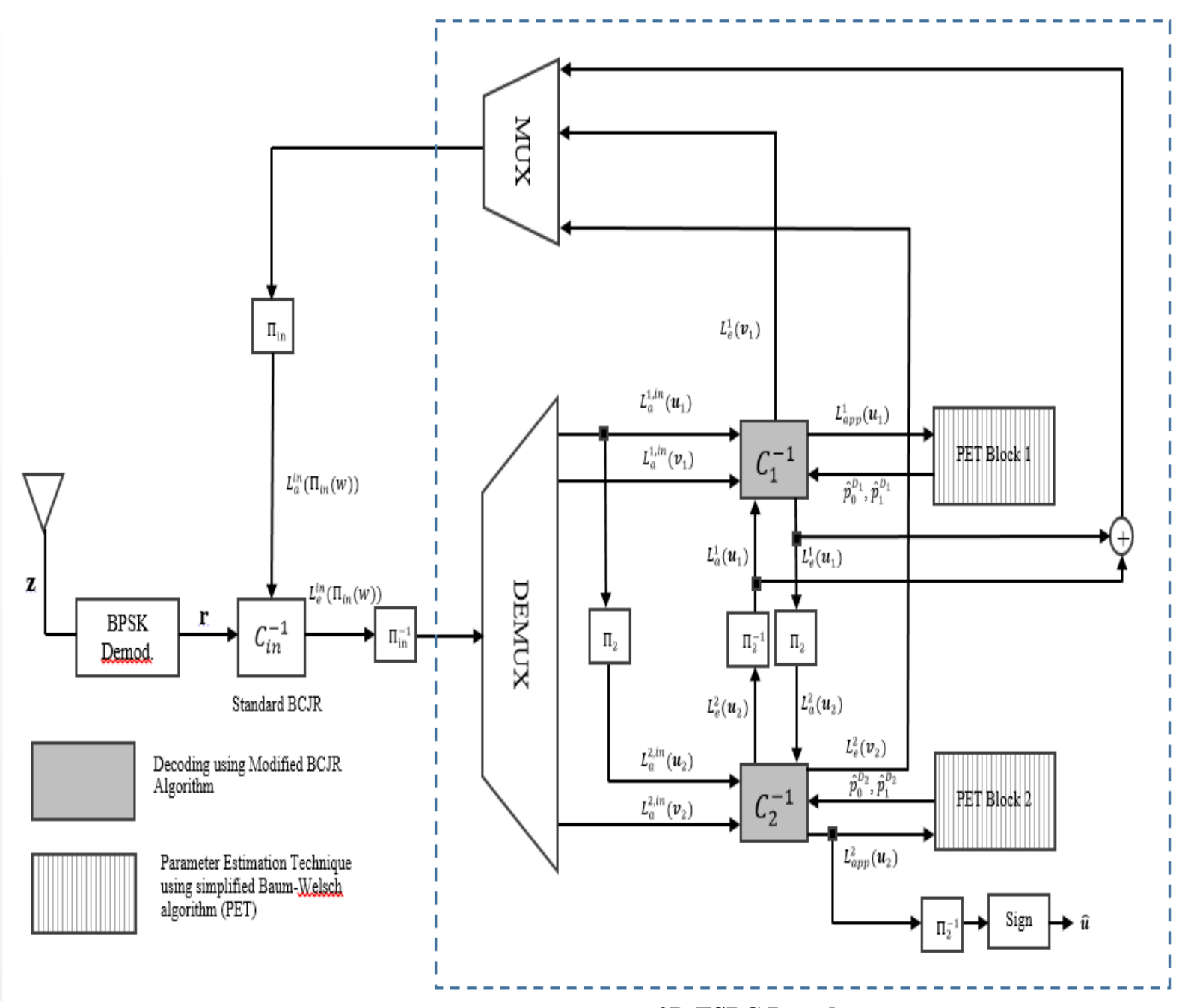

2D TSPC Decoder

Figure 3. The proposed design at the receiver for the 2D JSCC system with parameter estimation 


\section{RESULT AND ANALYSIS}

In this work, the main goal is to develop an accurate parameter estimation technique to estimate the unknown source statistics at the receiver for the 2D JSCC system performing as close as possible to the ideal 2D JSCC system as proposed in [9]. The BER performance is evaluated, by finding the number of errors of the decoded binary sequence from the original binary sequence from the source and this number of errors is divided by the frame length of the source sequence [28]. The Markov source was initialized with 0.5 to both dimensions. To illustrate the result, a simulation of BER against SNR simulation was performed using MATLAB software.The configuration used in the simulations to evaluate the performance of the proposed 2D JSCC system are given in Table 1. Turbo-Single Parity-Check-Code (TSPCC) was used as the outer code. The Single-Parity-Check (SPC) code consisting of 8 codeword length and 7 information bits is used for the component code of the outer code. The rate-1 Recursive-Systematic-Convolutional (RSC) code which had generator polynomial, $\left(G_{r}, G\right)=(3,2)_{8}$ (given in octal numeral system) is used in the inner code where $G_{r}$ is the feedback polynomial and $G$ is the feed-forward polynomial.

Figure 4 illustrates the comparison in BER performance of the proposed 2D JSCC system and the ideal 2D JSCC system with source correlation in horizontal and vertical direction varying from $=$ until $=$. The performance of 2D JSCC system assisting with the BWA-based estimation technique or also known as 2D JSCC system with Parameter Estimation 1 (2D-JSCC-PET1 system) is very close to the ideal 2D JSCC system and improves when the source correlation becomes stronger. For example, at BER level of, an improvement of $1.18 \mathrm{~dB}$ is achieved is achieved with the proposed 2D-JSCC-PET1 system over the non-JSCC system for $\mathrm{p}=0.7$ and the improvement increases to $4.48 \mathrm{~dB}$ for $\mathrm{p}=0.9$. The performance gain between the ideal 2D JSCC system and the proposed 2D-JSCC-PET1 system is about the same for each value of p. As can be seen in the Figure 4, the ideal 2D JSCC system is only slightly better than the proposed 2DJSCC-PET1 system. However, the ideal 2D JSCC system relies on the assumption of perfect source knowledge at the receiver, which may not be suitable practically. Usually, the parameter estimation technique requires complex computations concerning its accuracy that might lead to performance degradation but despite the degradation that may occur, the gain for our proposed 2D-JSCC-PET1 system is not too far from the ideal system and hence, ensuring the system performing better in terms of the accuracy and the reliability of the data transmission at the receiver.

In another simulation, the performance difference between the proposed 2D-JSCC-PET1 system and the similar system without any parameter estimation denoted as the 2D JSCC system reveals that without the estimation technique, the performanceof the system could not do any better for unknown source statistics. The gain is measured as a difference of Eb/NO at the BER level of between the 2D JSCC systems and the non-JSCC system. The performance gains of different systems are tabulated in Table 2.

Figure 5 compares the BER performance between the proposed 2D-JSCC-PET1 system and 2DJSCC-PET2 system when the correlation parameters are unknown for sources, exhibiting correlation varying from $=$ until $=$. The performance gain between the proposed 2D-JSCC-PET1 system and the similar 2D JSCC system using different parameter estimation or the Parameter Estimation 2 (PET2) is summarized in Table 3. Figure 5 indicates that the PET1 module is much better than the PET2 module which simply comparing the reliable extrinsic LLRs gathered from each decoder [11].

It can be seen from Table 3 that there is a significant difference in BER performance between the proposed 2D-JSCC-PET1 system and the 2D-JSCC-PET2 system which indicates that the proposed 2DJSCC-PET1 system is performing better than the 2D-JSCC-PET2 system. Based on the performance in Figure 5, it can be seen that the gain difference over the non-JSCC system between the proposed 2D-JSCCPET1 system and the 2D-JSCC-PET2 system becomes larger for stronger source correlation, where at $p=0.7$, the gain difference is $0.84 \mathrm{~dB}$ and it increases to $3.47 \mathrm{~dB}$ at $\mathrm{p}=0.9$. The performance achieved by the 2D JSCC systems with the parameter estimation technique show a significant difference when compared to the 2D JSCC system without employing any estimation method (the 2D JSCC system) and the non-JSCC system.

Table 1. Default Settings Used for the Simulations of the Proposed 2D JSCC System

\begin{tabular}{cc}
\hline Parameter & Value \\
\hline Outer Code & 2D TSPCC \\
Component Codes for Outer Code & SPC $(8,7)$ Code \\
Inner Code & Rate-1 RSC $(3,2) \mathrm{s}$ Code \\
Frame Length, $K_{U T}$ & $608 \times 608$ bits \\
$\Pi_{2}$ & Block Interleaver: $608 \times 608$ bits \\
$\Pi_{i n}$ & Random Interleaver : Length 363,888 bits \\
Code Rate, Rc & 0.78 \\
Decoding Iterations & 25 \\
\hline
\end{tabular}

Int J Elec \& Comp Eng, Vol. 8, No. 4, August 2018 : 2633 - 2642 


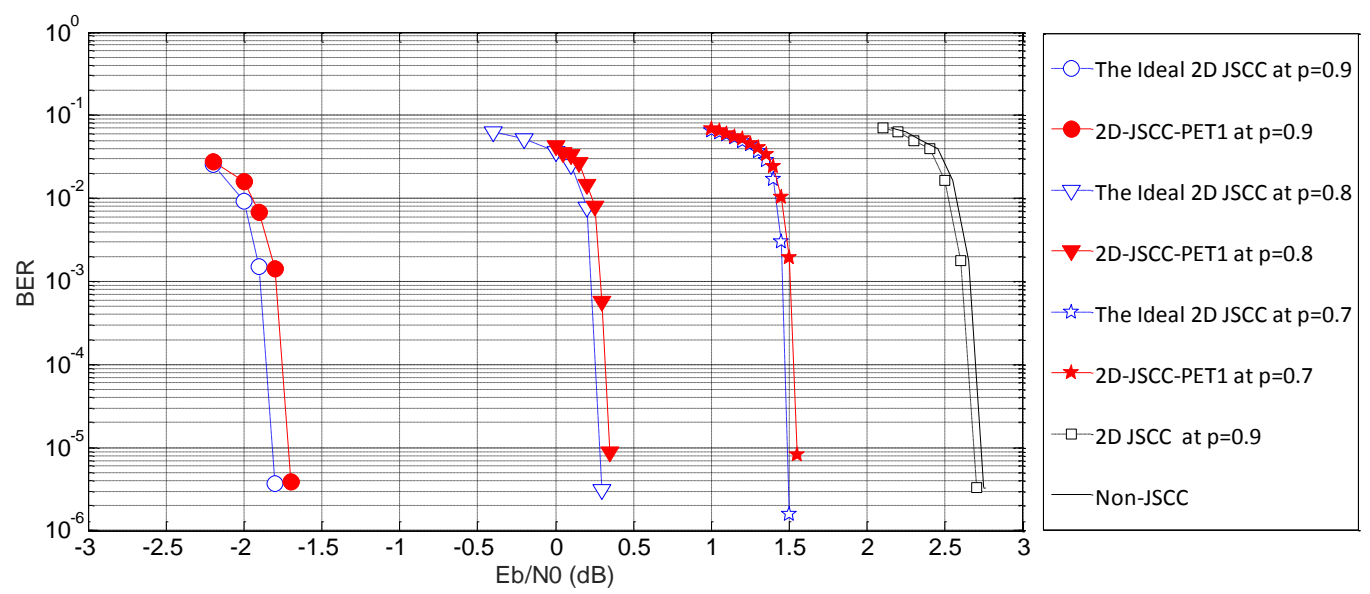

Figure 4. Comparison in BER performance of the proposed 2D-JSCC-PET1 system and the ideal 2D JSCC system [9] as the value of $\mathrm{p}$ increases

Table 2. Performance Gain in dB of the Proposed 2D-JSCC-PET1 System and the Ideal 2D JSCC System

\begin{tabular}{cccccc}
\multicolumn{7}{c}{ Over the non-JSCC System } \\
\hline$p$ & $\begin{array}{c}\text { The ideal 2D } \\
\text { JSCC system } \\
(\mathrm{dB})\end{array}$ & $\begin{array}{c}\text { 2D-JSCC- } \\
\text { PET1 } \\
(\mathrm{dB})\end{array}$ & $\begin{array}{c}\text { Gain for The ideal 2D } \\
\text { JSCC system } \\
(\mathrm{dB})\end{array}$ & $\begin{array}{c}\text { Gain for } \\
\text { 2D-JSCC- } \\
\text { PET1 (dB) }\end{array}$ & $\begin{array}{c}\text { Gain } \\
\text { Difference } \\
(\mathrm{dB})\end{array}$ \\
\hline 0.7 & 1.49 & 1.54 & 1.23 & 1.18 & 0.05 \\
0.8 & 0.29 & 0.34 & 2.43 & 2.38 & 0.05 \\
0.9 & -1.82 & -1.71 & 4.54 & 4.48 & 0.06 \\
\hline
\end{tabular}
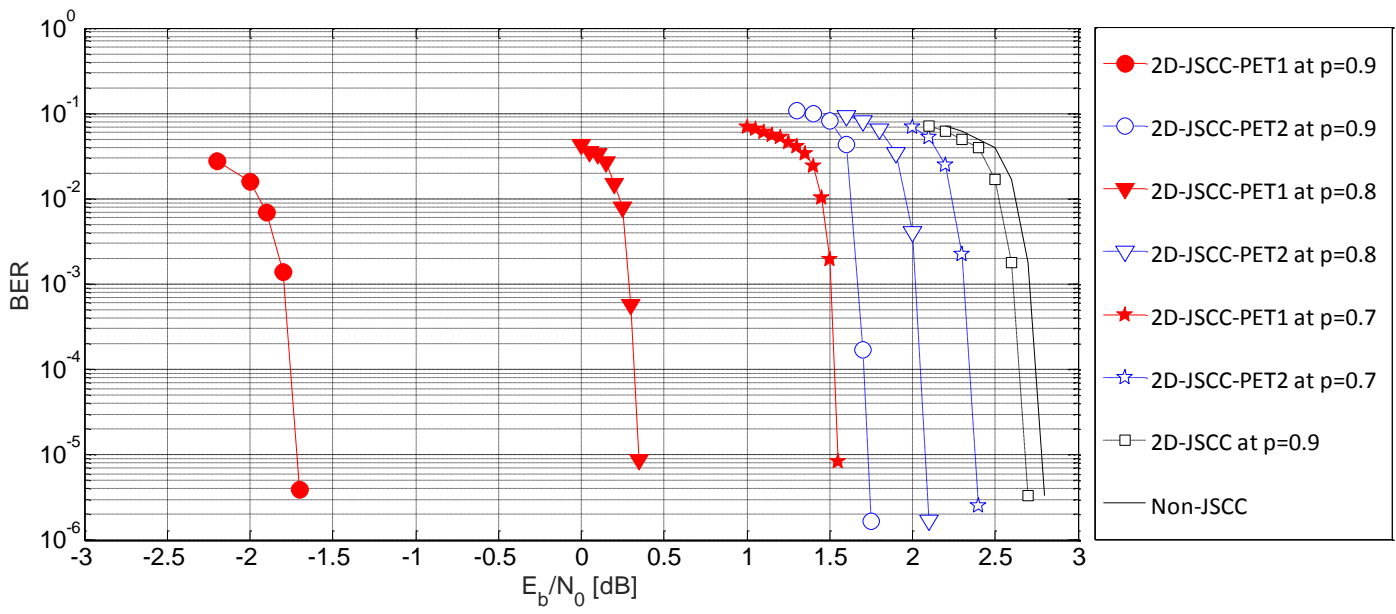

Figure 5. Performance gain in $\mathrm{dB}$ of the proposed 2D-JSCC-PET1 system and the 2D-JSCC-PET2 system

Table 3. Performance Gain in $\mathrm{dB}$ of the Proposed 2D-JSCC-PET1 System and the 2D-JSCC-PET2 System

\begin{tabular}{cccccc}
\multicolumn{5}{c}{ over the non-JSCC System } \\
\hline$p$ & $\begin{array}{c}\text { 2D-JSCC- } \\
\text { PET1 } \\
(\mathrm{dB})\end{array}$ & $\begin{array}{c}\text { 2D-JSCC-PET2 } \\
(\mathrm{dB})\end{array}$ & $\begin{array}{c}\text { Gain for 2D- } \\
\text { JSCC-PET1 } \\
(\mathrm{dB})\end{array}$ & Gain for 2D-JSCC-PET2 $(\mathrm{dB})$ & $\begin{array}{c}\text { Gain } \\
\text { Difference } \\
(\mathrm{dB})\end{array}$ \\
\hline 0.7 & 1.54 & 2.38 & 1.18 & 0.34 & 0.84 \\
0.8 & 0.34 & 2.06 & 2.38 & 0.64 & 1.74 \\
0.9 & -1.71 & 1.71 & 4.48 & 1.01 & 3.47 \\
\hline
\end{tabular}




\section{CONCLUSION AND FUTURE WORK}

This research proposed a 2D JSCC system exploiting estimation technique based on the BaumWelsh algorithm in the decoder on unknown source statistics (2D-JSCC-PET1). The BWA that is used in the proposed design has been detailed with its calculations and equations. For sources with identical, the gain of the proposed 2D-JSCC-PET1 system and the ideal 2D JSCC system over the non-JSCC system shows very small difference which are $4.54 \mathrm{~dB}$ and $4.48 \mathrm{~dB}$, respectively. The results also indicate that the difference in performance gain between of the proposed 2D-JSCC-PET1 system and the ideal 2D JSCC system is approximately the same for strongest source correlation. The difference of proposed 2D-JSCC-PET1 system and ideal 2D JSCC system values at $p=0.7$ and $p=0.9$ are slightly the same i.e. $0.05 \mathrm{~dB}$ and $0.06 \mathrm{~dB}$, respectively. This concludes that it is possible to get the best performance with the parameter estimation technique on unknown source correlation knowledge. The proposed 2D-JSCC-PET1 system is then compared to another estimation technique based on a previous study [11] known as 2D-JSCC-PET2 system. The difference in performance gain between the proposed 2D-JSCC-PET1 system and the 2D-JSCC-PET2 system increases with the increment of source correlation, i.e., from a difference of $0.84 \mathrm{~dB}$ at $p=0.7$ to a difference of $3.47 \mathrm{~dB}$ at $p=0.9$. Future studies could develop and enhance parameter estimation technique in 2D JSCC system with a higher order of Markov Source and higher dimension of source correlation in order to obtain a more reliable and better performance.

\section{ACKNOWLEDGEMENTS}

The author would like to thank the reviewers for the suggestions which help to improve the quality of this paper. In addition, the authors are also very thankful to UTM Razak School of Engineering and Advanced Technology, UTM Kuala Lumpur, Malaysia for providing resources.

\section{REFERENCES}

[1] Berrou, C., Glavieux, A. and Thitimajshima, P, "Near Shannon Limit Error Correcting Coding and Decoding: Turbo-Codes", Proceedings of IEEE I.S. International Conference on Communications (ICC), Geneva, Switzerland, pp. 1064-1070, 1993.

[2] Salim, Mohammad, et al. "A New Block S-Random Interleaver for Shorter Length Frames for Turbo Codes," Bulletin of Electrical Engineering and Informatics, vol. 2, no. 4, pp. 293-298, 2013.

[3] Wang, Jian, Jianping Li, and Chaoshi Cai, "A Novel Decoding Algorithm for BICM-ID Embedded Turbo Codes," Indonesian Journal of Electrical Engineering and Computer Science, vol. 12, no. 5, pp. 3460-3466, 2014.

[4] Kamruzzaman, M. M., "Performance Comparison of Turbo Coded Single Input Multiple Output System Using Selective Combining, Equal Gain Combining and Maximal Ratio Combining," International Journal of Information and Network Security, vol. 3, no. 2, p. 64, 2014.

[5] Shannon, C. E., "A Mathematical Theory of Communication", The Bell System Technical Journal, 27, pp. 379-423, 1948.

[6] Sayood, K. and Borkenhagen, J. C., "Use of Residual Redundancy in the Design of Joint Source/Channel Coders", IEEE Transactions on Communications, vol. 39, no. 6, pp. 839-846, 1991.

[7] Y. Huo, T. Wang, R. Maunder and L. Hanzo, "Two-Dimensional Iterative Source-Channel Decoding for Distributed Video Coding," in IEEE Communications Letters, vol. 18, no. 1, pp. 90-93, January 2014.

[8] Camiciotti, L., Lamy, C., Meilhac, L., Olivieri, S. and Verdi, P., "Joint Source Channel Coding for 4G Multimedia Streaming", Wireless World Research Forum (WWRF), Helsinki, Finland, 2001.

[9] Izhar, M.A.M Fisal, N Zhou, X Anwar,K Matsumoto, T ,"Exploitation of 2D binary source correlation using turbo block codes with fine-tuning", 7th International Symposium on Turbo Codes and Iterative Information Processing (ISTC), 2013.

[10] Izhar, M.A.M Fisal, N Zhou, X Anwar, K Matsumoto, "Utilization of 2-D Markov source correlation using block turbo codes", International Symposium on Turbo Codes and Iterative Information Processing, ISTC. 56-60. 2012.

[11] Y. Zhao, W. Zhong, and J. Garcia-Frias, "Transmission of correlated senders over a Rayleigh fading multiple access channel," Signal Process.,vol. 86, pp. 3150-3159, Nov. 2006.

[12] B. W. Khoueiry and M. R. Soleymani, "Joint channel estimation and raptor decoding over fading channel," 2014 27th Biennial Symposium on Communications (QBSC), Kingston, ON, 2014, pp. 168-172

[13] Qingxiong Deng, R. G. Machado and A. G. Klein, "Adaptive channel estimation in decode and forward relay networks," 2012 46th Annual Conference on Information Sciences and Systems (CISS), Princeton, NJ, 2012, pp. 1-6.

[14] W. Turin, "Unidirectional and parallel Baum-Welch algorithms," in IEEE Transactions on Speech and Audio Processing, vol. 6, no. 6, Nov 1998. pp. 516-523.

[15] Garcia-Frias, J., "Combining hidden Markov source models and parallel concatenated codes", IEEE Communications Letters, vol. 1 no. 4 1997, pp. 111-113.

[16] J. Garcia-Frias and J. D. Villasenor, "Turbo decoding of hidden Markov sources with unknown parameters," Data Compression Conference, 1998. DCC '98. Proceedings, Snowbird, UT, 1998, pp. 159-168. 
[17] J. Garcia-Frias and J. D. Villasenor, "Joint source channel coding and estimation of hidden Markov structures," Proceedings. 1998 IEEE International Symposium on Information Theory (Cat. No.98CH36252), Cambridge, MA, 1998 , pp. 201.

[18] J. Garcia-Frias, J. Villasenor, "Simplified methods for combining hidden Markov models and turbo codes", Proc. VTC'99, 1999, pp. 1580-1584.

[19] J. Garcia-Frias, J.D. Villasenor, "Joint turbo decoding and estimation of hidden Markov sources", Selected Areas in Communications IEEE Journal on, vol. 19, pp. 1671-1679, 2001, ISSN 0733-8716.

[20] Anwar, A.K. "Iterative Decoding and Channel Estimation over Hidden Markov Fading Channels", Faculty of the Virginia Polytechnic Institute and State University, 2000.

[21] Baum, L. E., Petrie, T., Soules, G. and Weiss, N., "A Maximization Technique Occurring in the Statistical Analysis of Probabilistic Functions of Markov Chains.” Ann. Math. Statist., 1970, vol. 41, no. 1, pp. 164-171.

[22] Dempster, A. P., Laird, N. M. and Rubin, D. B. "Maximum Likelihood from Incomplete Data via the EM Algorithm.” Journal of the Royal Statistical Society. Series B (Methodological), 1977, vol. 39, no. 1, pp. 1-38.

[23] L. Ping, S. C. and Yeung, K. L., "Iterative Decoding of Multi-dimensional Concatenated Single Parity Check Codes." Proceedings of IEEE International Conference on Communications. Atlanta, USA. 1998, pp. 131-135.

[24] Tee, J. S. K. and Taylor, D. P., "Multiple Parallel Concatenated Single Parity-check Codes", Proceedings of IEEE International Conference on Communications. Helsinki, Finland, 2001, pp. 60-64.

[25] Zhou, X., Anwar, K. and Matsumoto, T., "Serially Concatenated Joint Source-Channel Coding for Binary Markov Sources," 6th International ICST Conference on Communications and Networking (CHINACOM). Harbin, China. 2011.

[26] Zhou, X., Anwar, K. and Matsumoto, T., "EXIT Chart Based Joint Source Channel Coding for Binary Markov Sources," Proceedings of IEEE Vehicula Technology Conference (VTC Fall). Quebec City, Canada. 2012, pp. 1-5.

[27] Bahl, L., Cocke, J., Jelinek, F. and Raviv, J., "Optimal Decoding of Linear Codes for Minimizing Symbol Error Rates (Corresp.),” IEEE Transactions on Information Theory, 1974, vol. 20, no. 2, pp. 284-287.

[28] Daniel Jurafsky, James H. Martin, “Chapter 8: Hidden Markov Models, Speech and Language Processing”, Prentice Hall, 2014.

\section{BIOGRAPHIES OF AUTHORS}

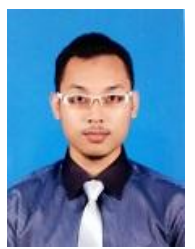

Muhammad Izzat Amir Mohd Nor received the B.Eng (Hons) in Electrical and Computer Engineering (Communication), International Islamic University Malaysia (IIUM - 2012). He is currently pursuing his Master of Philosophy at UTM Razak School of Engineering and Advanced Technology, Universiti Teknologi Malaysia (UTM) Kuala Lumpur. He is a member in various professional organizations such as Institute of Electrical and Electronics Engineers (IEEE) and The Institution of Engineers Malaysia (IEM). His research interests include channel coding, coding theory, joint source-channel coding, parameter estimation technique, IoT, wireless communication and cloud computing.

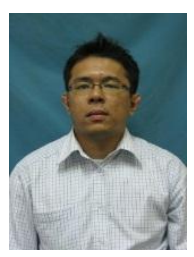

Mohd Azri Mohd Izhar received his M.Eng. degree in electrical engineering (communications) from the University of Sheffield, U.K., in 2008 where he received the Mappin medal award for outstanding academic performance and the Institute of Electrical and Electronics Engineers (IEEE) prize for the best communications-related final year project. He received the Ph.D. degree in electrical engineering from Universiti Teknologi Malaysia (UTM), Malaysia, in 2014. Since 2014, he has been a senior lecturer with UTM Kuala Lumpur campus. He was visiting the Southampton Wireless Group at the University of Southampton, U.K. for 2 years in 2015. His current research interests include channel coding, coding theory, joint source-channel coding, coded modulation, cooperative communications, cognitive radio, IoT and quantum communications.

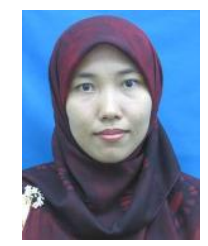

Norulhusna Ahmad graduated from Universiti Teknologi Malaysia (UTM) in 2001 with BSc in Electrical Engineering. She joined UTM as a staff and later pursuing her study at the same university. She received her Master degree of Electrical Engineering (Telecommunication) and PhD in Electrical Engineering in 2003 and 2014, respectively. Currently, she is a lecturer at Razak School of Science and Advanced Technology, UTM KL. During her PhD, she did an attachment in Japan Advanced Institute of Science and Technology (JAIST) under the supervision of Prof. Dr. Tadashi Matsumoto and Asst. Prof. Dr. Khoirul Anwar on the project in non-orthogonal frequency division multiplexing (n-OFDM) system. Her expertise is on the area of digital signal processing and wireless communication. Her research interests are on future communication such as $5 \mathrm{G}$ and cognitive radio focusing on error correcting codes, turbo equalization, OFDM, resource allocation, network coding and cooperative communication. 


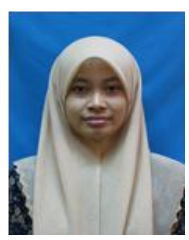

Universiti Teknologi Malaysia (UTM - 2006), the M.Sc. degree in Telecommunication and Information Engineering at Universiti Teknologi MARA (UiTM - 2008), and the Ph.D. degree from the Universiti Teknologi Malaysia (UTM - 2015). She is currently a senior lecturer at UTM Razak School of Engineering and Advanced Technology, Universiti Teknologi Malaysia Kuala Lumpur. She is a member in various professional organizations such as Institute of Electrical and Electronics Engineers (IEEE) and The Instituttion of Engineers Malaysia (IEM). She also a research member of Wireless Communication Centre (WCC) which is one of Higher Institution Centres of Excellence (HICoE) in Malaysia. Currently, WCC leads a research on Fifth Generation (5G). Her research interests include mobile and wireless communications, error control coding, relay networks, cooperative communications, Hybrid ARQ Cross Layer Design and iterative receiver. 